\title{
Insight into aroma attributes change during the hot-air-drying process of white shrimp using GC-MS, E-Nose and sensory analysis
}

\author{
Di ZHANG ${ }^{1}$ (D), Hong-Wu JI $I^{1,2,3,4,5 *}$, Gui-Xin LUO $^{1}$, Hao CHEN ${ }^{1}$, Shu-Cheng LIU ${ }^{1,2,3,4,5}$, Wei-Jie MAO
}

\begin{abstract}
Aroma attributes are one of the most important criteria that affect the flavor quality of dried shrimp, but the dynamic changes of aroma attributes remain largely unknown during the drying process. The present study investigated aroma attributes change during the hot-air-drying process of shrimp using gas chromatography-mass spectrometry (GC-MS), electronic nose (E-nose) and sensory analysis. The potential correlations among volatile compounds, sensory attributes and E-nose data were analyzed by partial-least-squares regression (PLSR). Results showed that the aroma characteristic of shrimps changed significantly during processing. The odor in the fresh shrimp was very light, and the key aroma compounds mainly consisted of trimethylamine and three aldehydes. The aroma characteristics mainly consisted of roasted and meat-like odors had come into being gradually with the decrease of water activity $\left(A_{w}\right)$, and the aroma attributes were the most acceptable at about $A_{w} 0.274$ (hot-air drying for $7 \mathrm{~h}$ ). Four kinds of aroma-active compounds (pyrazines, amines, aldehydes and heterocyclic compounds) made important contributions to the formation of aroma characteristics. The PLSR result showed a good correlation between most variables of volatile compounds, E-nose data and sensory attributes.
\end{abstract}

Keywords: white shrimp; hot-air-drying; aroma attributes; sensory analysis; electronic nose; gas chromatography-mass spectrometry.

Practical Application: The current research about aroma attributes during the hot-air-drying process of shrimps provides a theoretical basis for the control of flavor and quality of dried shrimp.

\section{Introduction}

Over the past decades, white shrimp (Penaeus vannamei), a high yield economic fishery resource, is widely consumed because of its nutritional values and attractive flavor (Cheok et al., 2017; Kleekayai et al., 2016). Dried shrimp is highly appreciated by consumers for their distinctive aroma, which develops upon the heating process (Cheok et al., 2017; Chung et al., 2019; Mall \& Schieberle, 2016, 2017; Zhang et al., 2020a). The drying process is the main step in the production of dried shrimp and also considered as an important step in the formation of the characteristic aroma (Souza \& Bragagnolo, 2014; Tachihara et al., 2004; Zhang et al., 2020a). Shrimp aroma determines the individuality of dried shrimp products and is one of the most important criteria to evaluate the quality (Lu et al., 2011; Souza \& Bragagnolo, 2014; Zhang et al., 2020a). It is well-known that fresh shrimp have little odor, generally showing a faint grassy, seawater-like odor. After the drying process, dried shrimp produce a characteristic shrimp aroma. N-containing heterocycles, trimethylamine, S-containing compounds and common carbonyl compounds were reported to make contributions to the formation of shrimp-like aroma (Mall \& Schieberle, 2016, 2017; Okabe et al., 2019; Rochat et al., 2009; Zhang et al., 2020b). Up to now, most of the studies mainly focused on the composition of volatile compounds in dried shrimp products, whereas the dynamic changes of aroma attributes during the drying process in dried shrimp products are still not well understood.

Currently, the sensory evaluation of shrimp aroma is still the main method in the shrimp industry. The methodology can be applied to describe various attributes of food samples by recording word descriptions and sensory intensities of trained assessors. Nevertheless, there are some deficiencies in sensory evaluation, e.g., human preference, time-consuming and variability (Calanche et al., 2019; Chen et al., 2019). Aroma components of shrimp products have also been traditionally analyzed using gas chromatography-mass spectrometry (GC-MS). Volatile compounds can be effectively identified to confirm the source of aroma attributes using GC-MS. However, analysis and interpretation of complex data are very time consuming and do not always lead in the correct direction due to the univariate methods and the inherent low selectivity of GC-MS (Gallegos et al., 2017). As a potential alternative to traditional techniques, electronic-nose (E-nose) technology is gaining popularity in the analysis of volatiles. In the last few decades, E-nose has experienced rapid development and played a tremendous role in many fields. The 
instrument is equipped with an array of metal oxide microbalance sensors, where each element responds to the sensed chemical (Chung et al., 2019; Feng et al., 2011; Chen et al., 2018). In recent years, combined applications of GC-MS, sensory evaluation and E-nose technology in shrimp products were widely reported by several studies (Kleekayai et al., 2016; Zhang et al., 2020b). However, it is still a challenge to match valuable information on the volatiles with sensory attributes.

Multivariate statistical tools, including partial-least-squares regression (PLSR) and principal component analysis (PCA), have been specifically designed for the visualization and analysis of complex sets in different samples (Granato et al., 2018; Zielinski et al., 2020). In many papers, multivariate statistical analysis was widely used to reveal the relationship between the chemical data and sensory attributes, and to identify those chemical components that have an important effect on the overall flavor (Miyazaki et al., 2012; Qin et al., 2013; Viljanen et al., 2014). Previous studies have reported the aroma characterization of shrimp products using sensory analysis, E-nose and GC-MS; however, only limited comprehensive studies have dealt with the correlation between sensory analysis and GC-MS analysis or E-nose data (Rochat et al., 2009; Zhang et al., 2020b). There is no report about the correlation of sensory attributes, GC-MS and E-nose data analysis regarding aroma attributes of dried shrimp products.

This study aimed to analyze aroma attributes change of white shrimp during the hot-air-drying process using sensory analysis, E-nose and GC-MS. The key active-aroma compounds were identified by odor activity value (OAV), and the potential correlations between volatile components, E-nose data and sensory attributes were analyzed by analysis of PLSR. These may provide information in-depth to enhance our understanding of the mechanisms on aroma formation during the hot-air drying process of shrimp.

\section{Materials and methods}

\subsection{Materials}

Raw fresh shrimp (Penaeus vannamei, 8-10 cm average length) were purchased at a local supermarket (Zhanjiang, China). Shrimp were covered with ice water to keep them alive, and transported to the laboratory within $1 \mathrm{~h}$. The component of the raw shrimp was protein $(17.56 \pm 0.47) \%$, moisture $(74.82 \pm 1.59) \%$, fat $(2.75 \pm 0.23) \%$, sugar $(0.87 \pm 0.06) \%$ and ash $(3.52 \pm 0.47) \%$.

\subsection{Preparation of shrimp samples}

Fresh shrimp were killed using crushed ice. Raw shrimp (not peeling) were drained and placed in a hollow metal plate. Shrimp were dried using an Eyela NDO-710 electrothermostatic blast oven (Tokyo Rikakikai Co. Ltd., Tokyo, Japan). The samples were hot-air dried for $0,1,2,3,4,5,6,7,8$ and $9 \mathrm{~h}$ at a constant relative humidity of ca. $20 \%$ and an air temperature of $85^{\circ} \mathrm{C}$. Shrimps were uniformly distributed in in hollow metal plates $\left(15 \mathrm{~kg} / \mathrm{m}^{2}\right)$. Each shrimp was flipped every $30 \mathrm{~min}$ during the hot-air drying, to ensure both sides heat evenly. Each shrimp sample was frozen with liquid nitrogen and minced to a fine powder. Water activity $\left(A_{w}\right)$ in shrimps at different drying time was determined at $25^{\circ} \mathrm{C}$ using a Decagon Aqua Lab meter (Pullman, WA, USA) according to the method of Okpala (2015).

\subsection{Sensory analysis}

The sensory analysis was performed by the quantitative description analysis method. The sensory panel consisted of 11 experienced panelists from Guangdong Ocean University (Guangdong, China), who were well trained according to the ISO standard 8556:2012 (International Organization for Standardization, 2012). These panels showed accumulated sufficient experience and score accuracy for each aroma descriptor after training. A common description vocabulary was generated to characterize aroma attributes, and the characteristic descriptors of shrimp samples were quantified using six sensory descriptors (fishy, smoky/burnt, sweet, caramel, roasted/nutty and cooked-meat-like). The intensity scale was ranked on a scale from 0 (not perceivable) to 5 (strongly perceivable) in steps of 0.1 (Zhang et al., 2020a). The descriptors were compared with aqueous solutions of the following reference odorants (Zhang et al., 2018): fishy ((Z)-4-heptenal), smoky/burnt (2-methoxyphenol), cooked-meat-like (3-(methylthio)propionaldehyde), sweet (maltol), roasted/nutty (2,5-dimethylpyrazine) and caramel (4-hydroxy-2,5-dimethyl-3(2H)-furanone). For aroma profile analyses, five grams of each sample was weighed into a sealed bottle coded with three digit codes in a random order to prevent bias and equilibrated for $30 \mathrm{~min}$ in a water bath at $60{ }^{\circ} \mathrm{C}$. The evaluation was carried out at room temperature and one at a time, with a 5 min wait between samples.

\subsection{Electronic-nose analysis}

The E-nose analysis was performed according to the procedure described by Chen et al. (2018) with some modifications. A commercial PEN3 E-nose system (WinMuster Airsense Analytics Inc., Schwerin, Germany) was used to acquire data on the volatiles. Sensors of PEN3 E-nose respond to representative sensitive compounds (Melucci et al., 2016). Briefly, before detection, $4 \mathrm{~g}$ of each sample that came from the same specimen of GC-MS analysis was placed in a $25 \mathrm{~mL}$ glass bottle, then capped with a PTFE silicone stopper. After that, the headspace of the sample was equilibrated at room temperature $\left(25^{\circ} \mathrm{C}\right)$ for $20 \mathrm{~min}$, which could avoid sensor drift caused by environmental changes. The measurement phase lasted for $60 \mathrm{~s}$, and the interval for data collection was $1 \mathrm{~s}$. In this work, only the stable values of sensors were used for further data analysis. Each test was performed for three samples and every sample was replicated at least five times until relatively stable results were obtained.

The measured data were analyzed using PCA with the WinMuster software of the E-nose system. Sensor response values obtained from the E-nose were preconditioned with the standard normal variate to eliminate signal drift (Zhu et al., 2019).

\subsection{Volatile compounds extracted using solid-phase microextraction (SPME)}

Extraction of volatile compounds was performed according to Zhang et al. (2020a). The SPME fiber coated with divinylbenzene/ 
carboxen/polydimethylsiloxane (DVB/CAR/PDMS, 50/30 $\mu \mathrm{m}$ ) (Supelco, Bellefonte, PA, USA) was employed to extract volatile compounds in shrimp samples. The fiber was inserted into the headspace of a $25 \mathrm{~mL}$ glass vial that contained $2 \mathrm{~g}$ of sample and $2 \mu \mathrm{L}$ of methyl nonanoate (1.632 g/L in $n$-pentane). Samples were exposed to an SPME fiber with equilibration in a water bath at $65^{\circ} \mathrm{C}$ for $40 \mathrm{~min}$. After extraction, the fiber was desorbed at $240{ }^{\circ} \mathrm{C}$ for $4 \mathrm{~min}$ in the GC-MS injector in splitless mode.

\subsection{GC-MS analysis}

The GC-MS analysis was performed on a QP2010-Plus GC-MS instrument (Shimadzu, Tokyo, Japan). A DB-WAX capillary column $(30 \mathrm{~m} \times 0.25 \mathrm{~mm} \times 0.25 \mu \mathrm{m}$; Agilent, Santa Clara, CA, USA) was employed. The initial oven temperature was $40{ }^{\circ} \mathrm{C}$ maintained for $3 \mathrm{~min}$, then $40-100{ }^{\circ} \mathrm{C}$ at a rate of $5^{\circ} \mathrm{C} / \mathrm{min}$, then $100-180^{\circ} \mathrm{C}$ at $2^{\circ} \mathrm{C} / \mathrm{min}$, then raised to $250^{\circ} \mathrm{C}$ at $10^{\circ} \mathrm{C} / \mathrm{min}$ and held there for $5 \mathrm{~min}$. The carrier gas was helium (99.999\% purity) at a constant flow of $1.2 \mathrm{~mL} / \mathrm{min}$. The mass spectrometer had a mass range of $\mathrm{m} / z 30$ to 500 at a scanning rate of $1.8 \mathrm{~s}^{-1}$. The electron ionization mode was used with an electron impact energy of $70 \mathrm{eV}$. Ion source temperature and interface temperature were set at $230^{\circ} \mathrm{C}$ and $250^{\circ} \mathrm{C}$, respectively.

\subsection{Identification and quantitative analysis of volatile compounds}

The identification of volatile compounds was carried out by comparing the recorded mass spectra with the Wiley version 6.0 database (Wiley, Chichester, UK) and the NIST 2.0 MS libraries, retention index (RI) and comparing previous literature and published index data. The RIs were calculated from all of the volatile compounds using a C5-C25 n-alkanes series (SigmaAldrich Trading Co., Ltd., Shanghai, China), and the values were compared, when available, with values reported in the literature for similar chromatographic columns.

The internal standard (IS) method was used to quantify the volatile compounds. The mean value of triplicates was calculated using the following formula, odorant concentration = (compound peak area $\times$ IS concentration)/IS peak area ( $\mathrm{Pu}$ et al., 2019; Zhang et al., 2020b). The contribution of each odor to the overall fruit aroma was evaluated by the OAV, which was measured as the ratio of the concentration of each compound to its detection threshold in water. The threshold values were taken from information available according to Gemert (2011).

\subsection{Statistical analysis}

Data analysis was performed using SPSS, version 19 software (SPSS Inc., Chicago, IL, USA). All experiments were performed three times, and mean values were reported. Differences between groups were declared significant at $p<0.05$ by ANOVA with Duncan's test.

The correlations between volatile compounds, E-nose data and aroma attributes during the hot-air-drying process of shrimp were analyzed using PLSR through the Unscrambler version 9.7 (CAMO ASA, Oslo, Norway). All variables, such as volatile compounds, E-nose data and sensory scores were centered and standardized (1/Sdev) before applying PLS analyses and PLSR models were validated using full cross-validation.

\section{Results and discussion}

\subsection{Sensory evaluation}

Sensory evaluation is a reliable technology to directly reflect the characteristics and quality of food, and can translate color, odor, taste and texture into visual data (Calanche et al., 2019; Castilhos et al., 2019). A spider plot was created to observe the dynamic changes of aroma profile during the hot-air-drying process of shrimp (Figure 1).

Fresh shrimp had little odor. The score of fishy in S0 was the highest (1.1), followed by sweet (0.6), and scores of other aroma attributes were low. During the early period ( $0-2 \mathrm{~h})$, there was no obvious change in sweet, fishy, caramel and smoky/burnt odors while scores of roast/nutty and cooked-meat-like odors increased slightly, which indicated that the overall aroma of shrimp changed little at the inception stage. During the hot-air drying for 2-7 h, scores of six aroma attributes increased significantly $(p<0.05)$ compared with S0. In particular, scores of roast/nutty and cookedmeat-like odors were much stronger than other aroma attributes, which indicated that they were the main aroma characteristics of shrimp in the middle time of hot-air drying. During the late period (7-9 h), scores of most aroma attributes changed little. It was worth noting that the score of smoky/burnt odor in S8 and S9 increased significantly $(p<0.05)$ compared with that of S7. The overall aromas of S8 and S9 due to the addition of smoky/ burnt odor were unacceptable. The aroma attributes as a whole showed S7 were much better than the others.

\subsection{Water activity}

Water is an important medium for various chemical reactions, and most of the flavor precursors are water-soluble, so $A_{w}$ played an important role in the formation of aroma compounds. Previous research had reported that the meat-like flavor components were mainly derived from Maillard reaction, and the water activity could qualitatively affect the kind and amount of major volatiles produced during heating, which made contributions to

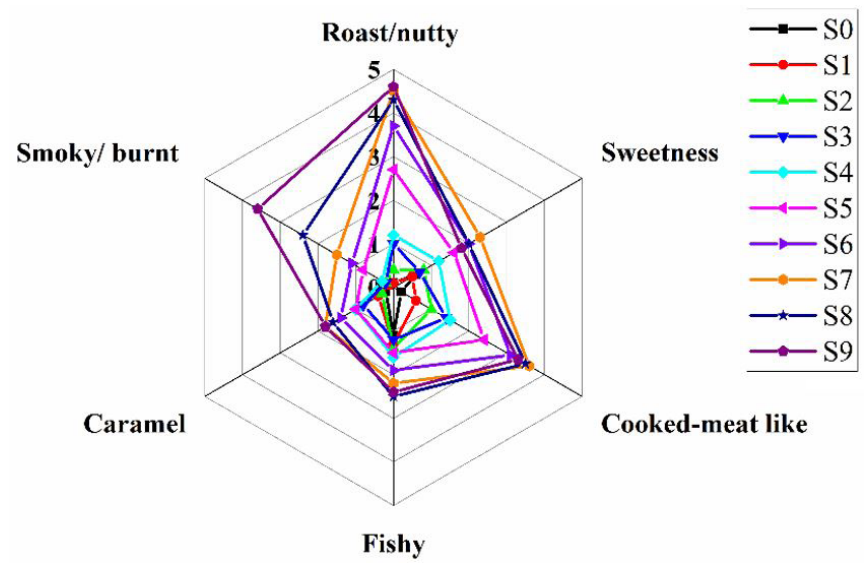

Figure 1. Radar map of aroma profiles during the hot-air-drying process of shrimp. S0, S1, S2, S3, S4, S5, S6, S7, S8 and S9 represent hot-air-dried shrimp for 0 h, 1 h, 2 h, 3 h, 4 h, 5 h, 6 h, 7 h, 8 h and 9 h, respectively. 
the formation of aroma characteristics (Hartman et al., 1984). Figure 2 showed that the $A_{w}$ change in shrimps with the drying time. At the early process $(0-2 \mathrm{~h})$, the $A_{w}$ of shrimps decreased slowly from 0.971 to 0.916 . At the middle process $(2-7 \mathrm{~h})$, the $A_{w}$ decreased rapidly from 0.916 to 0.274 . At the late process (7-9 h), the $A_{w}$ changed little (0.274-0.255). According to the result of sensory evaluation, the aroma attributes of shrimps at $7 \mathrm{~h}$ were considered as the most acceptable during drying process, meanwhile, the $A_{w} 0.274$ at $7 \mathrm{~h}$ was appropriate for long time storage of dried shrimp. Therefore, the stage could be used as the optimal condition for the aroma formation.

\subsection{E-nose analysis}

E-nose response to shrimp samples' aroma during the hot-airdrying process

The aroma characteristics of shrimps during at different drying time were analyzed using E-nose equipped with ten sensors,

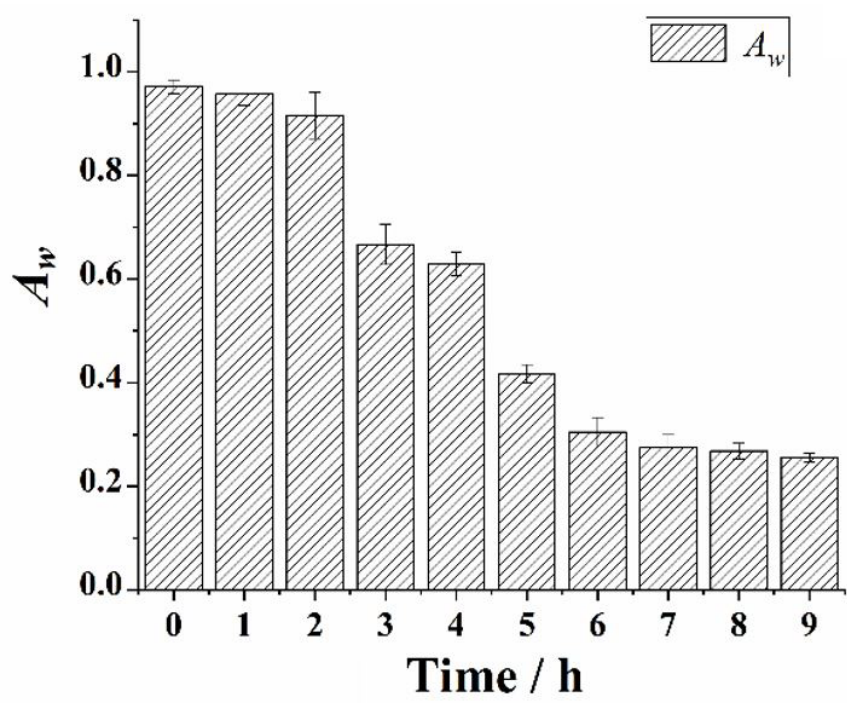

Figure 2. The $A_{w}$ change in shrimps with the drying time.
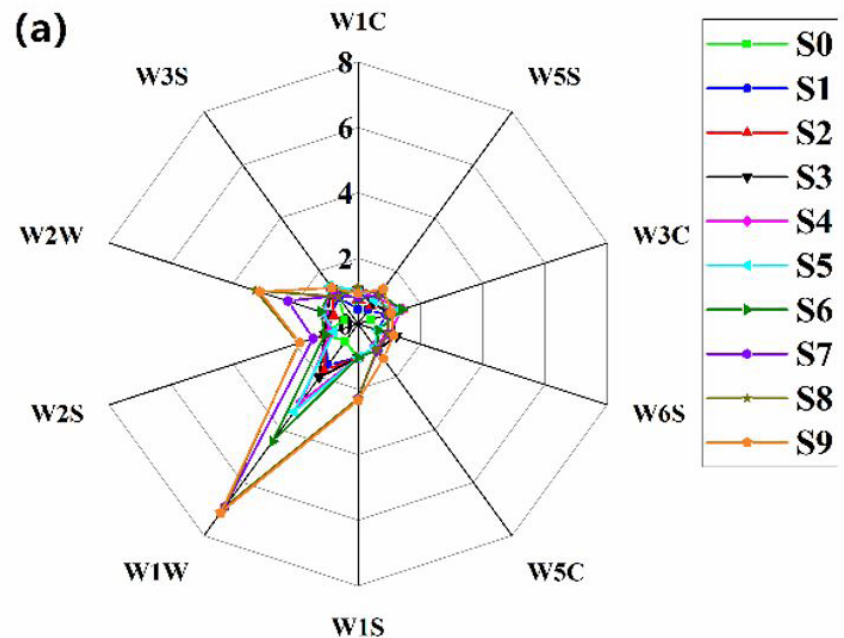

which depended on not only the concentration of molecules in odors, but also what odor molecules consist of Chen et al. (2018). Figure 3a shows polar graphs of the responses of the sensors to the 10 shrimp samples during the hot-air-drying process. Fresh shrimp samples (S0) represented a low response on all sensors, which indicated that fresh shrimp had little odor. During the early process $(0-2 \mathrm{~h})$, most of the sensors in S1 and S2 changed little while sensor W1W and sensor W3C increased a bit compared with S0. Radar chart shapes changed as the hotair drying proceeded (2-7 h), it was observed that responses of all sensors increased to different extents in S2 to S7, especially sensor W1W, sensor W2W, sensor W1S and sensor W2S. This indicated that an abundance of aroma compounds was produced in shrimp during the late stage of the hot-air-drying process. In addition, the radar chart shapes of S7, S8 and S9 were quite close, which suggested that these three samples might have similar aroma attributes during the late period (7-9 h).

Classification of aroma attributes of shrimp during the hot-airdrying process using PCA

Principal component analysis is a statistical technique for the reduction of input data dimension and is largely used for feature extraction. It captures the relevant information in a set of input data providing a lower dimension (Fernandes et al., 2019; Nascimento et al., 2020). For improved visualization of the data, PCA was performed to distinguish aroma attributes of 10 shrimp during the hot-air-drying process (Figure $3 \mathrm{~b}$ ). The contribution of the first two PCs (PC1 and PC2) reflects the completeness of the variable information based on $\mathrm{PC} 1$ and PC2. Most data points of shrimp samples (S0, S1 and S2) were distributed in the third quadrants, which indicated that aroma attributes of shrimp have not substantially changed at an early stage of hot-air drying. During the middle period (2-7 h), data points of shrimp samples were distributed in the first and second quadrants, which suggested that aroma attributes of shrimp were obviously different from fresh shrimp at this stage. In particular, data points of shrimp samples (S7, S8 and S9) were distributed in

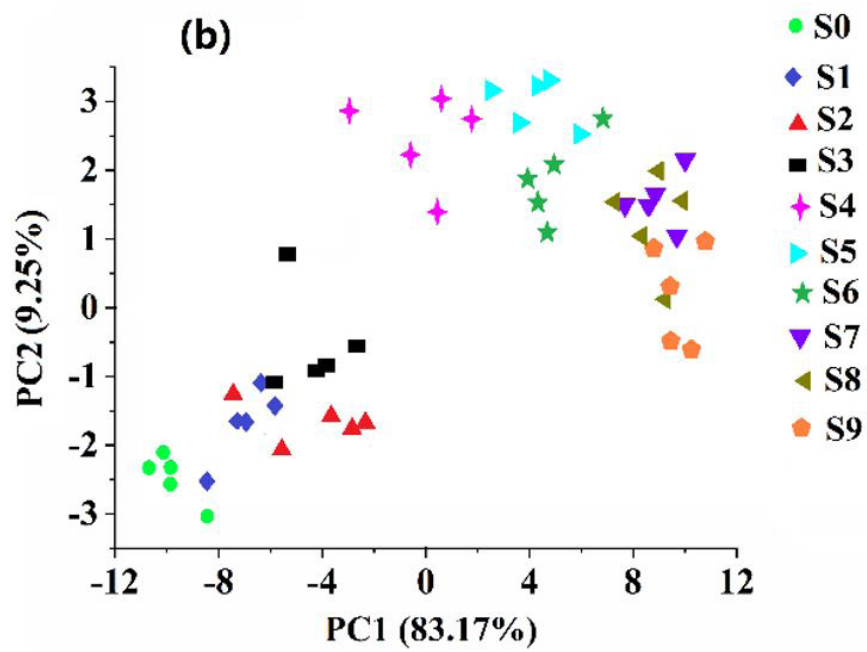

Figure 3. Radar map (a) and PCA analysis (b) of the E-nose during the hot-air-drying process of shrimp. S0, S1, S2, S3, S4, S5, S6, S7, S8 and S9 represent hot-air-dried shrimp for $0 \mathrm{~h}, 1 \mathrm{~h}, 2 \mathrm{~h}, 3 \mathrm{~h}, 4 \mathrm{~h}, 5 \mathrm{~h}, 6 \mathrm{~h}, 7 \mathrm{~h}, 8 \mathrm{~h}$ and $9 \mathrm{~h}$, respectively. 
a fairly small band, and these three samples might have similar aroma attributes. According to the GC-MS results, an abundance of aroma compounds was produced during the hot-air-drying process for 2-7 h, which may dramatically change the overall aroma of shrimp. E-nose analysis was consistent with the result of sensory evaluation.

\subsection{GC-MS analysis}

Shrimp collected during the hot-air-drying process were analyzed using GC-MS to illuminate the dynamic changes of volatile compounds. A total of 79 volatile compounds were identified and quantified, including two S-containing compounds, 15 pyrazines, 16 ketones, 17 hydrocarbons, three amines, 10 alcohols, three heterocyclic compounds, nine aldehydes and four esters (Table 1). As a whole, species and contents of volatile compounds varied greatly during the process. During the hotair drying for 0-9 h, pyrazines, hydrocarbons and heterocyclic compounds were the three most variable compounds. In the fresh shrimp, hydrocarbons, ketones and aldehydes were the most abundant compounds, which accounted for $94.06 \%$. During the hot-air drying for 0-2 $\mathrm{h}$, most of the compounds in S1 and S2 were comparatively close to fresh shrimp while a few pyrazines were produced (lower than $10 \mathrm{ng} / \mathrm{g}$ ), the content of ketones decreased significantly $(p<0.05)$, and the content of amines increased significantly $(p<0.05)$. During the hot-air drying for 2-7 $\mathrm{h}$, the kind and number of volatile compounds in shrimp samples increased drastically from $1667.73 \mathrm{ng} / \mathrm{g}$ (S2) to $17891.31 \mathrm{ng} / \mathrm{g}$ (S9). At this stage, pyrazines, ketones, amines, aldehydes, S-containing compound and heterocyclic compounds increased rapidly. Most of these compounds have low thresholds, and they contributed to the aroma characteristics in hot-air-dried shrimp. At the late period (7-9 h), large amounts of S-containing compounds and heterocyclic compounds were detected, but the number of volatile compounds changed little.

OAVs of volatile compounds in the hot-air-dried shrimp were calculated to identify the contributors to the aroma profile. Volatile compounds with $\mathrm{OAV} \geq 1$ (based on the published odor thresholds determined in water) are identified as aroma-active compounds (AACs), and AACs in shrimps at different drying time were shown in Table 2. About 4, 4, 2, 5, 7, 11, 16, 16, 17 and 15 kinds of AACs were identified at 0 h-, 1 h-, 2 h-, 3 h-, 4 h-, 5 h-, 6 h-, 7 h-, 8 h- and 9 h-dried samples, respectively. Drying process of shrimps was divided into three periods according to the AACs changes, early period: $0-2 \mathrm{~h}$, middle period: $2-7 \mathrm{~h}$, and late period: $7-9 \mathrm{~h}$.

\section{Early period (0-2 h)}

In fresh shrimp (S0), OAV summation was only 21.41, and trimethylamine, benzaldehyde, caproicaldehyde and amylaldehyde were the main AACs (accounting for 95.07\%). Trimethylamine comprised $60.04 \%$ of the total OAVs in fresh shrimp, and the main source of the odors. During the hot-air drying for $0-2 \mathrm{~h}$, OAV summation increased slightly, and the OAV summations of AACs in S1 and S2 were 43.31 and 93.45, respectively. However, the composition of AACs in the two samples changed little compared with $\mathrm{S} 0$, the main sources in AACs were mainly of rapidly increasing trimethylamine (accounted for 74.31-92.61\% of the total), with a few pyrazines (accounted for 7.45-20.24\%).

\section{Middle period (2-7 h)}

The content and amount of AACs changed significantly during this period, and the OAV summation increased rapidly from 93.45 (S2) to 752.91 (S7). These changes were mainly attributed to the increase of pyrazines, amines, aldehydes, and heterocyclic compounds by more than $85 \%$ in the OAV increment.

It is reported that pyrazines are important aroma compounds in shrimp products subjected to drying or heating treatment (Neethling et al., 2016; Tachihara et al., 2004; Zhang et al., 2020a). Pyrazines are derived from the Maillard reaction, which commonly have popcorn, peanut, roasted, and meat-like odors. Pyrazines' OAV showed the fastest increase, and reached to (549.79) at hot-air drying for $7 \mathrm{~h}$ (S7), which were increased by 86.15 times compared with S2. 3-Ethyl-2,5-dimethylpyrazine, 2,5-dimethylpyrazine, 2,3,5-trimethylpyrazine and 2-methyl-3,5diethylpyrazine were the highest OAV AACs in S7, which made important contributions to the formation of aroma attributes.

Amines are mainly produced from degradation of $\mathrm{N}$-containing organic compounds (Gu et al., 2013; Fan et al., 2017). Trimethylamine was the only amine in AACs, which could be found in all samples. It is very common in seafood products and usually regarded as a reduction product of trimethylamine oxide. Trimethylamine contributes to fishy and seawater-like odors according to previous papers (Mall \& Schieberle, 2016; Zhang et al., 2020b). Though OAVs of trimethylamine represented a substantial increase as the hot-air drying proceeded $(2-7 \mathrm{~h})$, the proportion in total constantly decreased from $74.30 \%$ to $22.26 \%$.

Aldehydes are important volatile flavor compounds in aquatic products, and produced from the deamination of amino acids. OAVs of benzaldehyde and 3-(methylthio)propionaldehyde obviously increased as the hot-air drying proceeded, and OAV summation of the two compounds accounted for $6.44 \%$ of the total in S7. 3-(methylthio)propionaldehyde contributed to cooked-meat-like and onion odors, and benzaldehyde has an unpleasant almond odor.

Heterocyclic compounds are well-known as the common flavor components in the thermal treatment of meats and aquatic products. Furan compounds are important heterocyclic compounds and generally contribute to milk, cooked-meat-like, fat and roasted potato flavors (Shahidi, 1998; Zhang et al., 2019, 2020a). During the middle period, two heterocyclic compounds in AACs were identified as 2-pentylfuran and pyridine. Pyridine contributed to an unpleasant odor and had a negative effect on the aroma attributes. In general, the hydrocarbons, phenols, alcohols, acids and esters are generally considered to make little contribution because of their high odor thresholds.

\section{Late period (7-9h)}

It is observed that compositions and contents of AACs during this period were close, OAV summations were 752.91 (S7), 844.59 (S8) and 896.95 (S9), and they had 12 AACs in common. Four volatile compounds (3-(methylthio)propionaldehyde, trimethylamine, 


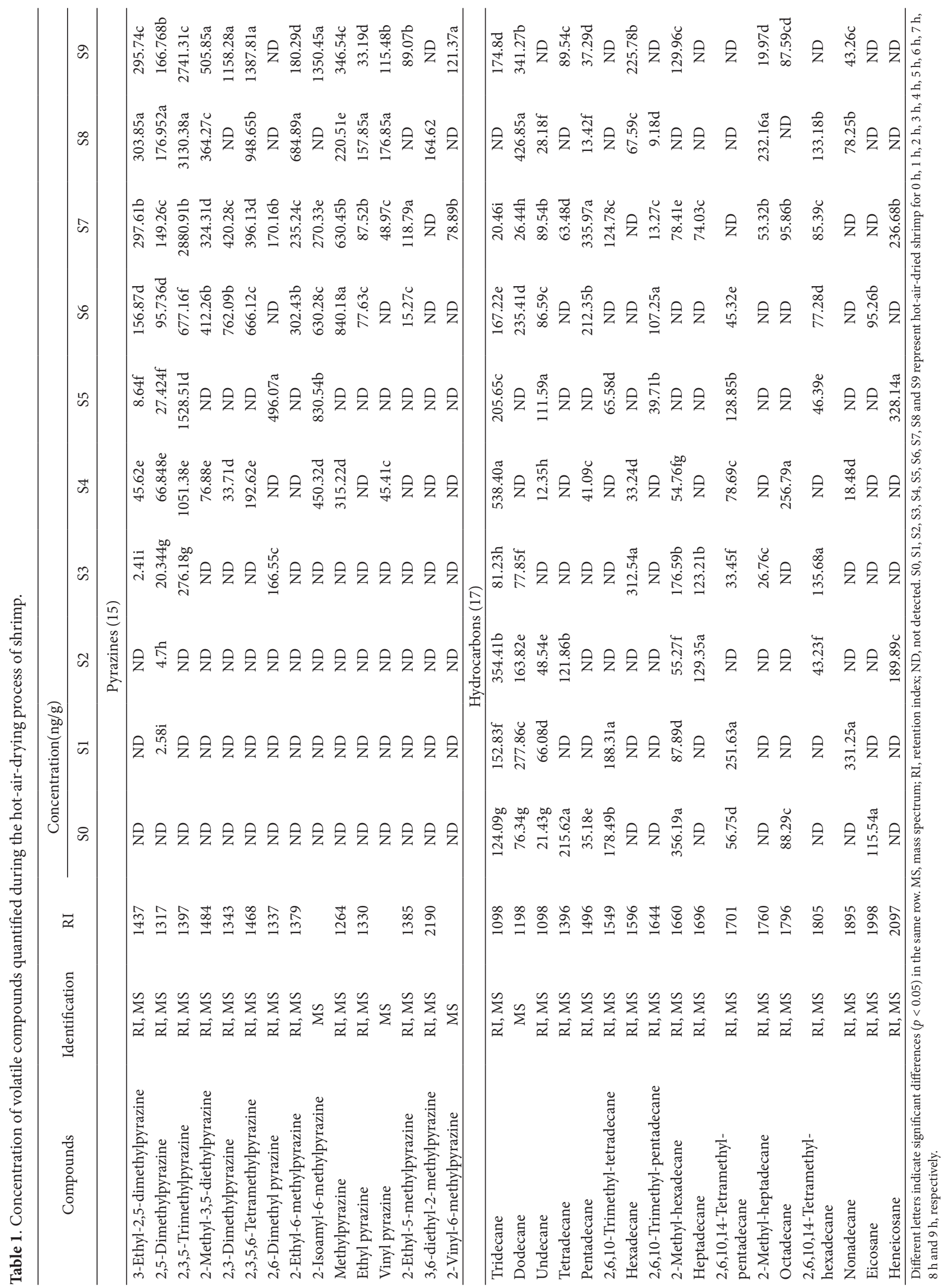




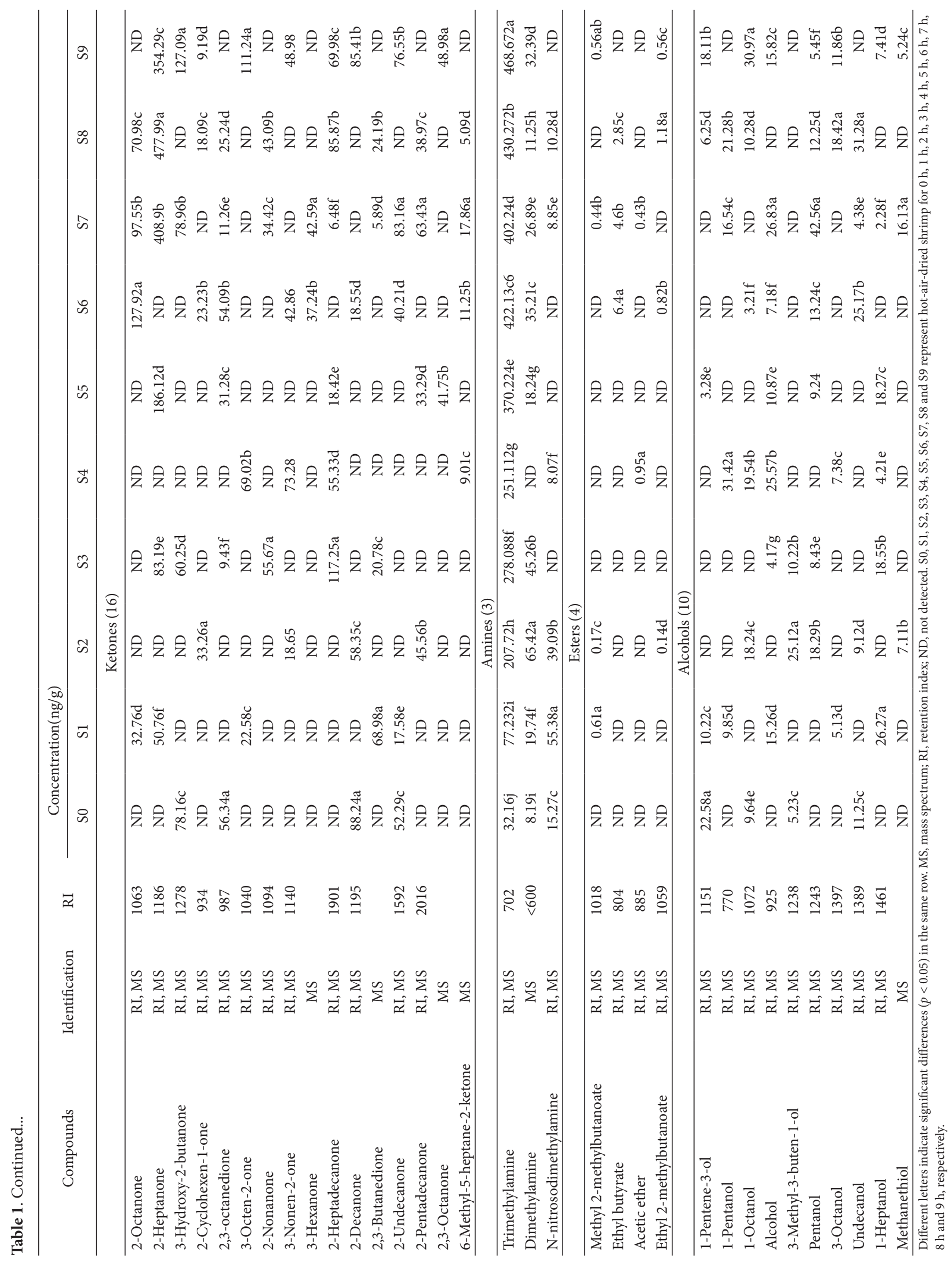




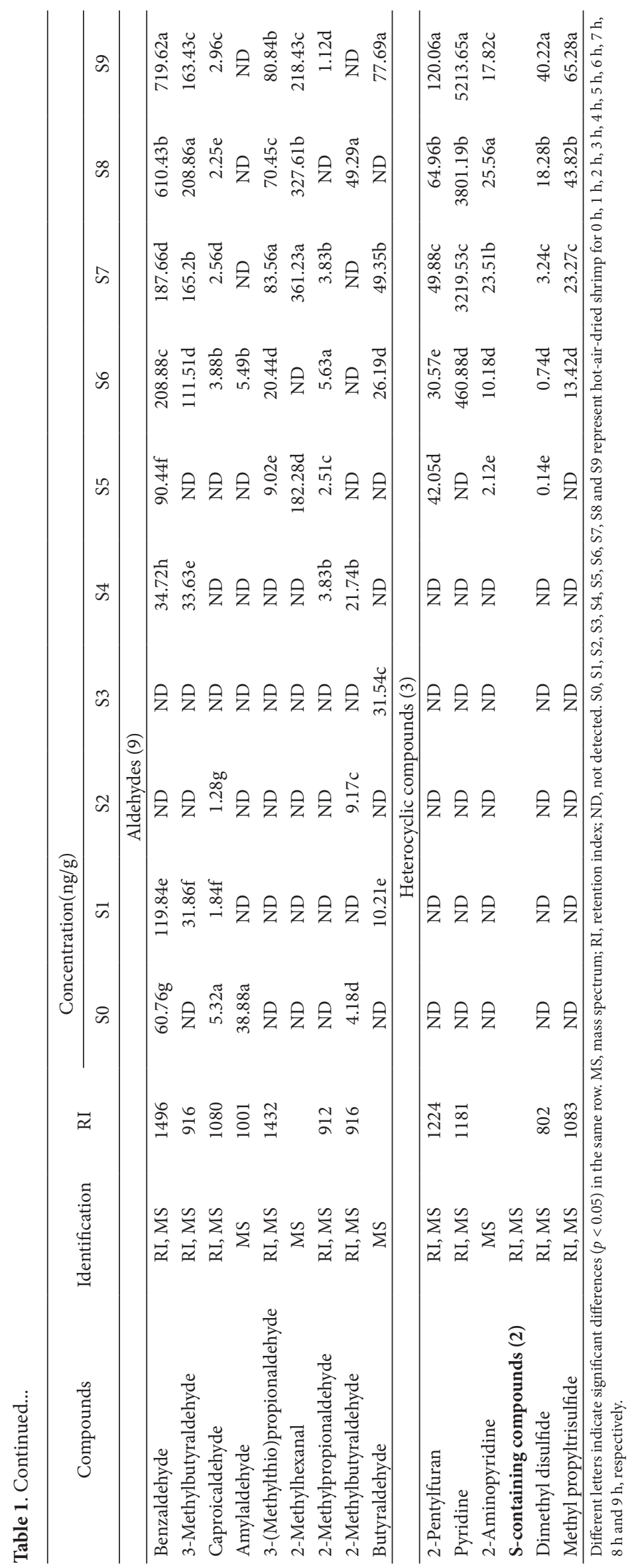




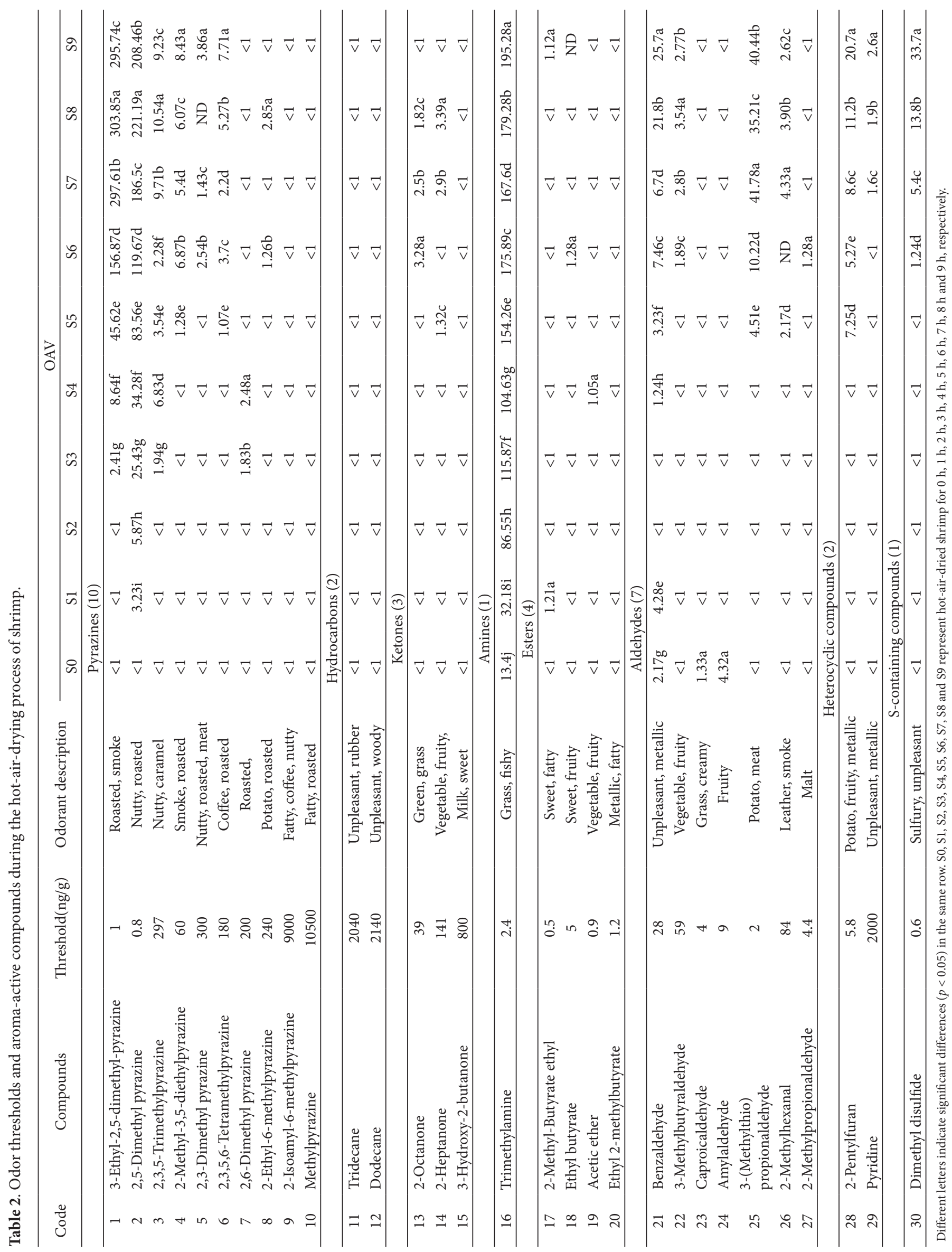


2,5-dimethylpyrazine and 3-ethyl-2,5-dimethylpyrazine) were the highest AACs of the three samples, and they accounted for more than $85 \%$ of the total. This indicated that S7, S8 and S9 had similar aroma attributes. It is worth noting that OAVs of benzaldehyde in S8 and S9 increased by 2.25 and 2.84 times that of S7, respectively. Benzaldehyde has an unpleasant almond and smoky odors, and too high concentration would lead to the deterioration of the overall aroma (Cai et al., 2016). This phenomenon explained the reason for the increase of smoky odor during the late period. GC-MS results were consistent with results of sensory evaluation and E-nose.

In conclusion, aroma compounds of shrimps apparently changed during the hot-air drying process. The middle period (2-7 h) was the crucial period for the development of aroma characteristic of dried shrimps, which gradually converted trimethylamine and aldehydes of fresh shrimps to pyrazines, amines, aldehydes and heterocyclic compounds of dried shrimps.

\subsection{Correlation between GC-MS results, E-nose data and sensory attributes}

In an attempt to study the relationships between sensory attributes, E-nose data and volatile compounds, PLSR models were performed. As shown in Figure 4a, the X-matrix was projected as volatile compounds with thresholds; the Y-matrix was projected as six sensory properties (roasted/nutty, smoky/burnt, sweet, cooked-meat-like, fishy and caramel). The derived PLSR model explained $82 \%$ of the variance in $\mathrm{X}$ and $86 \%$ of the variance in Y. The inner ellipse showed that $50 \%$ of the explained variance and the outer ellipse showed $100 \%$ of the explained variance (Kovács et al., 2010). Most of the sensory attributes and volatile compounds were located between the small and big ellipses, except methylpyrazine, tridecane, dodecane, 3-hydroxy-2-butanone, acetic ether and 2-methylpropionaldehyde. 3-Ethyl-2,5-dimethylpyrazine, 2,5-dimethylpyrazine, 2,3,5-trimethylpyrazine, 2-methyl-
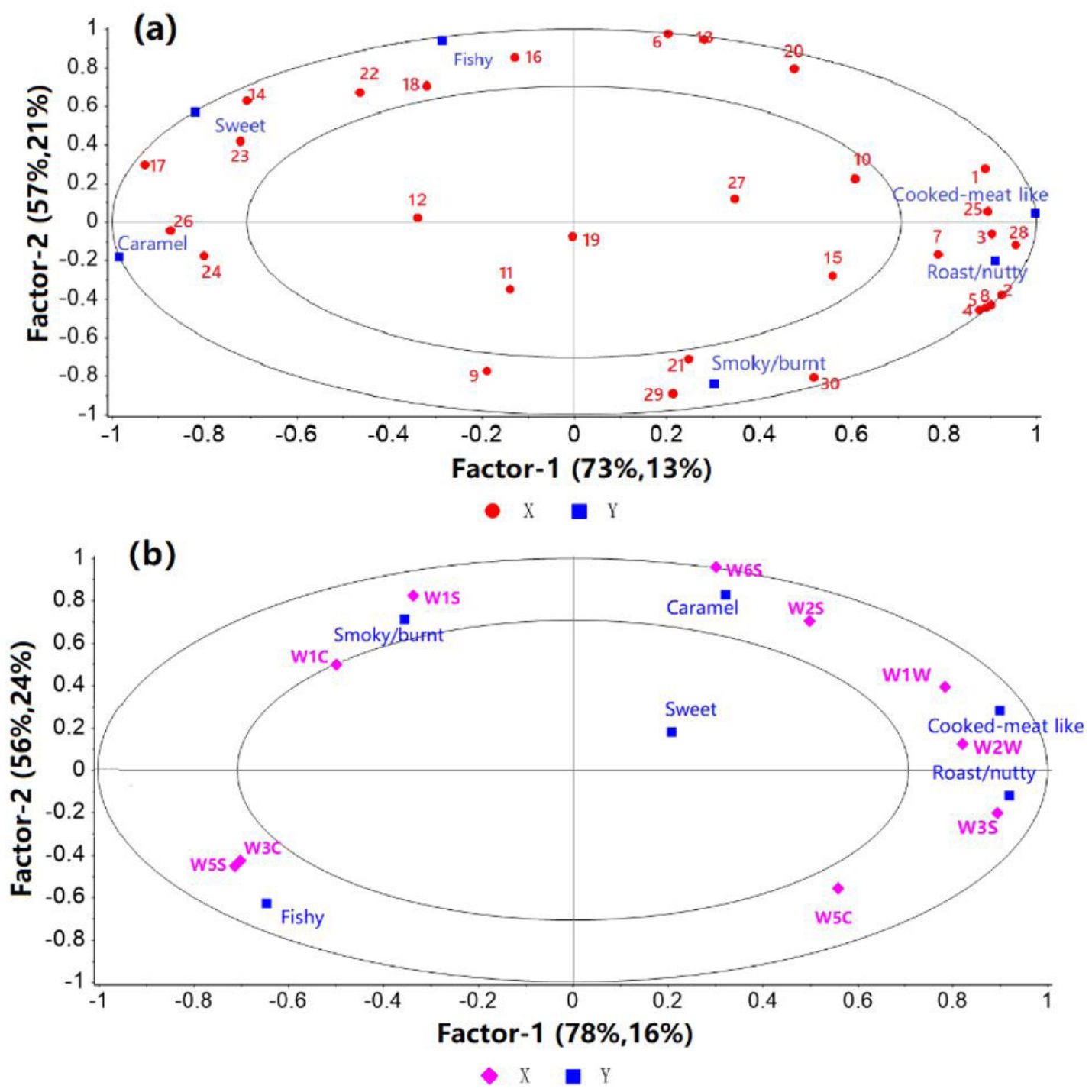

Figure 4. PLSR correlation loadings plot of indicator variables of volatile compounds, E-nose data and sensory evaluation (1-30 denote the 30 compounds defined in Table 2) during the hot-air-drying process of shrimp. (a) The model was derived from volatile compounds as the X-matrix (red point) and sensory attributes as the Y-matrix (blue point); (b) The model was derived from the signals of the E-nose as the X-matrix (purple point) and sensory attributes as the Y-matrix (blue point). The small and big ellipses represent $R^{2}=50$ and $100 \%$, respectively. 
3,5-diethylpyrazine, 2,3-dimethylpyrazine, 2,6-dimethylpyrazine, 2-ethyl-6-methylpyrazine, 3-(methylthio)propionaldehyde and 2-pentylfuran had correlations with cooked-meat-like and roasted/nutty odors. Trimethylamine, ethyl butyrate and 3-methylbutyraldehyde have correlations with fishy odor. 2-Heptanone, ethyl 2-methylbutyrate and caproicaldehyde showed positive correlations with sweet odors. Amylaldehyde and 2-methylhexanal have correlations with caramel odor. Benzaldehyde, pyridine and dimethyl disulfide have correlations with smoky/burnt odor. PLSR were consistent with results of sensory evaluation and AACs.

In Figure $4 \mathrm{~b}$, the $\mathrm{X}$-matrix was designed as the signal values from the E-nose and designated as independent variables, six sensory properties were designated as dependent variables. The derived PLSR model included two significant PCs explaining most of the E-nose data and sensory attributes, except sweet odor. Variables of W1W, W2W and W3S from the X-matrix have correlations with roasted/nutty and cooked-meat-like odors from the Y-matrix. Variables of W5S and W3C showed positive correlations with a fishy odor. Variables of W6S, W2W and W2S showed positive correlations with caramel odor. Variables of W1C and W1S showed a good correlation with smoky/burnt odor. PLSR were consistent with results of E-nose, sensory evaluation and AACs.

\section{Conclusions}

The aroma characteristics of shrimps changed significantly during the hot-air-drying process. Along with hot-air drying, the aroma intensity in shrimp increased while the aroma characteristics including mainly roasted and meat-like odors had come into being gradually. In shrimp with $A_{w} 0.274$ (hot-air drying for $7 \mathrm{~h}$ ), the number of AACs increased to 16, and the aroma attributes as a whole were much better than the others. Four kinds of AACs made important contributions, namely, pyrazines, amines, aldehydes and heterocyclic compounds. The results will provide a theoretical basis for the control of flavor and quality of hot-air-dried shrimp during the drying process.

\section{References}

Cai, L., Li, D., Dong, Z., Cao, A., Lin, H., \& Li, J. (2016). Change regularity of the characteristics of Maillard reaction products derived from xylose and Chinese shrimp waste hydrolysates. LebensmittelWissenschaft + Technologie, 65, 908-916. http://dx.doi.org/10.1016/j. lwt.2015.09.007.

Calanche, J., Tomas, A., Martinez, S., Jover, M., Alonso, V., Roncales, P., \& Beltran, J. A. (2019). Relation of quality and sensory perception with changes in free amino acids of thawed seabream (Sparus aurata). Food Research International, 119, 126-134. http://dx.doi. org/10.1016/j.foodres.2019.01.050. PMid:30884640.

Castilhos, M. B. M., Del Bianchi, V. L., Gomez-Alonso, S., GarciaRomero, E., \& Hermosin-Gutierrez, I. (2019). Sensory descriptive and comprehensive GC-MS as suitable tools to characterize the effects of alternative wine making procedures on wine aroma. Part I: BRS Carmem and BRS Violeta. Food Chemistry, 272, 462-470. http://dx.doi.org/10.1016/j.foodchem.2018.08.066. PMid:30309569.

Chen, Q., Song, J., Bi, J., Meng, X., \& Wu, X. (2018). Characterization of volatile profile from ten different varieties of Chinese jujubes by HS-SPME/GC-MS coupled with E-nose. Food Research International,
105, 605-615. http://dx.doi.org/10.1016/j.foodres.2017.11.054. PMid:29433254.

Chen, X. H., Chen, D. J., Jiang, H., Sun, H. Y., Zhang, C., Zhao, H., Li, X., Yan, F., Chen, C., \& Xu, Z. (2019). Aroma characterization of Hanzhong black tea (Camellia sinensis) using solid phase extraction coupled with gas chromatography-mass spectrometry and olfactometry and sensory analysis. Food Chemistry, 274, 130-136. http://dx.doi. org/10.1016/j.foodchem.2018.08.124. PMid:30372917.

Cheok, C. Y., Sobhi, B., Mohd Adzahan, N., Bakar, J., Abdul Rahman, R., Ab Karim, M. S., \& Ghazali, Z. (2017). Physicochemical properties and volatile profile of chili shrimp paste as affected by irradiation and heat. Food Chemistry, 216, 10-18. http://dx.doi.org/10.1016/j. foodchem.2016.08.011. PMid:27596386.

Chung, N., Ameer, K., Jo, Y., \& Kwon, J. H. (2019). Comparison of electronic sensing techniques for screening dried shrimps irradiated using three types of approved radiation with standard analytical methods. Food Chemistry, 286, 395-404. http://dx.doi.org/10.1016/j. foodchem.2019.02.038. PMid:30827624.

Fan, Y., Yin, L., Xue, Y., Li, Z., Hou, H., \& Xue, C. (2017). Analyzing the flavor compounds in Chinese traditional fermented shrimp pastes by HS-SPME-GC/MS and electronic nose. Journal of Ocean University of China, 16(2), 311-318. http://dx.doi.org/10.1007/ s11802-017-3194-y.

Feng, T., Zhuang, H., Ye, R., Jin, Z., Xu, X., \& Xie, Z. (2011). Analysis of volatile compounds of Mesona Blumes gum/rice extrudates via GC-MS and electronic nose. Sensors and Actuators. B, Chemical, 160(1), 964-973. http://dx.doi.org/10.1016/j.snb.2011.09.013.

Fernandes, M. D. F. L., Moraes, S. M. D., Sousa, P. H. M. D., Magalhães, C. E. D. C., Almeida, M. M. B., \& Silva, M. G. D. V. (2019). Characterization of leaves used in infusion preparation grown in northeastern Brazil by chemometric methods based on their multielemental composition. Food Science and Technology, 39(Suppl. 1), 309-315. http://dx.doi.org/10.1590/fst.00718.

Gallegos, J., Arce, C., Jordano, R., Arce, L., \& Medina, L. M. (2017). Target identification of volatile metabolites to allow the differentiation of lactic acid bacteria by gas chromatography-ion mobility spectrometry. Food Chemistry, 220, 362-370. http://dx.doi. org/10.1016/j.foodchem.2016.10.022. PMid:27855912.

Gemert, L. J. (2011). Compilations of odour threshold values in air, water \& other media and compilations of flavour threshold values in water \& other media (2nd ed.). Georgia: Leffingwell \& Associates Canton.

Granato, D., Putnik, P., Kovačević, D. B., Santos, J. S., Calado, V., Rocha, R. S., Cruz, A. G. D., Jarvis, B., Rodionova, O. Y., \& Pomerantsev, A. (2018). Trends in chemometrics: Food authentication, microbiology, and effects of processing. Comprehensive Reviews in Food Science and Food Safety, 17(3), 663-677. http://dx.doi.org/10.1111/15414337.12341. PMid:33350122.

Gu, S. Q., Wang, X. C., Tao, N. P., \& Wu, N. (2013). Characterization of volatile compounds in different edible parts of steamed Chinese mitten crab (Eriocheir sinensis). Food Research International, 54(1), 81-92. http://dx.doi.org/10.1016/j.foodres.2013.05.018.

Hartman, G. J., Scheide, J. D., \& Ho, C. T. (1984). Effect of water activity on the major volatileś produced in a model system approximating cooked meat. Journal of Food Science, 49(2), 607-613. http://dx.doi. org/10.1111/j.1365-2621.1984.tb12480.x.

International Organization for Standardization - ISO. (2012). ISO 8586:2012: sensory analysis: general guidelines for the selection, training and monitoring of selected assessors and expert sensory assessors. Geneva: ISO.

Kleekayai, T., Pinitklang, S., Laohakunjit, N., \& Suntornsuk, W. (2016). Volatile components and sensory characteristics of Thai traditional 
fermented shrimp pastes during fermentation periods. Journal of Food Science and Technology, 53(3), 1399-1410. http://dx.doi. org/10.1007/s13197-015-2142-3. PMid:27570264.

Kovács, Z., Dalmadi, I., Lukács, L., Sipos, L., Szántai-Kőhegyi, K., Kókai, Z., \& Fekete, A. (2010). Geographical origin identification of pure Sri Lanka tea infusions with electronic nose, electronic tongue and sensory profile analysis. Journal of Chemometrics, 24(3-4), 121-130. http://dx.doi.org/10.1002/cem.1280.

Lu, F., Zhang, J. Y., Liu, S. L., Wang, Y., \& Ding, Y. T. (2011). Chemical, microbiological and sensory changes of dried Acetes chinensis during accelerated storage. Food Chemistry, 127(1), 159-168. http://dx.doi. org/10.1016/j.foodchem.2010.12.120.

Mall, V., \& Schieberle, P. (2016). Characterization of key aroma compounds in raw and thermally processed prawns and thermally processed lobsters by application of aroma extract dilution analysis. Journal of Agricultural and Food Chemistry, 64(33), 6433-6442. http://dx.doi. org/10.1021/acs.jafc.6b02728. PMid:27486834.

Mall, V., \& Schieberle, P. (2017). Evaluation of key aroma compounds in processed prawns (Whiteleg Shrimp) by quantitation and aroma recombination experiments. Journal of Agricultural and Food Chemistry, 65(13), 2776-2783. http://dx.doi.org/10.1021/acs. jafc.7b00636. PMid:28282986.

Melucci, D., Bendini, A., Tesini, F., Barbieri, S., Zappi, A., Vichi, S., Conte, L., \& Gallina Toschi, T. (2016). Rapid direct analysis to discriminate geographic origin of extra virgin olive oils by flash gas chromatography electronic nose and chemometrics. Food Chemistry, 204, 263-273. http://dx.doi.org/10.1016/j.foodchem.2016.02.131. PMid:26988501.

Miyazaki, T., Plotto, A., Baldwin, E. A., Reyes-De-Corcuera, J. I., \& Gmitter, F. G. Jr. (2012). Aroma characterization of tangerine hybrids by gas-chromatography-olfactometry and sensory evaluation. Journal of the Science of Food and Agriculture, 92(4), 727-735. http://dx.doi. org/10.1002/jsfa.4663. PMid:22413143.

Nascimento, M. M., Jesus, R. M. D., Santos, H. M., Silva, A. L. S. D. Jr., Campos, N. M. C. O., Silva, E. G. P. D., \& Lobo, I. P. (2020). Quality pattern evaluation of frozen soursop pulps: an assessment based on chemical composition and chemometric analysis. Food Science and Technology, 40(2), 508-516. http://dx.doi.org/10.1590/fst.04919.

Neethling, J., Hoffman, L. C., \& Muller, M. (2016). Factors influencing the flavour of game meat: a review. Meat Science, 113, 139-153. http://dx.doi.org/10.1016/j.meatsci.2015.11.022. PMid:26658009.

Okabe, Y., Inoue, Y., Kanda, Y., \& Katsumata, T. (2019). Odor-active compounds contributing to the characteristic aroma of shrimp cooked whole, including shells and viscera. European Food Research and Technology, 245(1), 233-241. http://dx.doi.org/10.1007/s00217018-3156-7.

Okpala, C. O. R. (2015). The physicochemical changes of farm-raised Pacific white shrimp (Litopenaeus vannamei) as influenced by iced storage. Food and Nutrition Sciences, 6(10), 906-922. http://dx.doi. org/10.4236/fns.2015.610095.

Pu, D., Zhang, H., Zhang, Y., Sun, B., Ren, F., \& Chen, H. (2019). Characterization of the key aroma compounds in white bread by aroma extract dilution analysis, quantitation, and sensory evaluation experiments. Journal of Food Processing and Preservation, 43(5), e13933. http://dx.doi.org/10.1111/jfpp.13933.
Qin, Z., Pang, X., Chen, D., Cheng, H., Hu, X., \& Wu, J. (2013). Evaluation of Chinese tea by the electronic nose and gas chromatography-mass spectrometry: Correlation with sensory properties and classification according to grade level. Food Research International, 53(2), 864-874. http://dx.doi.org/10.1016/j.foodres.2013.02.005.

Rochat, S., Egger, J., \& Chaintreau, A. (2009). Strategy for the identification of key odorants: application to shrimp aroma. Journal of Chromatography. A, 1216(36), 6424-6432. http://dx.doi. org/10.1016/j.chroma.2009.07.014. PMid:19651413.

Shahidi, F. (1998). Flavor of meat, meat products and seafoods (2nd ed.). London: Blackie Academic \& Professional.

Souza, H. A. L., \& Bragagnolo, N. (2014). New method for the extraction of volatile lipid oxidation products from shrimp by headspace-solidphase microextraction gas chromatography-mass spectrometry and evaluation of the effect of salting and drying. Journal of Agricultural and Food Chemistry, 62(3), 590-599. http://dx.doi.org/10.1021/ jf404270f. PMid:24354556.

Tachihara, T., Ishizaki, S., Ishikawa, M., \& Kitahara, T. (2004). Studies on the volatile compounds of roasted spotted shrimp. Chemistry \& Biodiversity, 1(12), 2024-2033. http://dx.doi.org/10.1002/ cbdv.200490155. PMid:17191837.

Viljanen, K., Heiniö, R.-L., Juvonen, R., Kössö, T., \& Puupponen-Pimiä, R. (2014). Relation of sensory perception with chemical composition of bioprocessed lingonberry. Food Chemistry, 157, 148-156. http:// dx.doi.org/10.1016/j.foodchem.2014.02.030. PMid:24679764.

Zhang, D., Ji, H., Liu, S., \& Gao, J. (2020a). Similarity of aroma attributes in hot-air-dried shrimp (Penaeus vannamei) and its different parts using sensory analysis and GC-MS. Food Research International, 137, 109517. http://dx.doi.org/10.1016/j.foodres.2020.109517. PMid:33233151.

Zhang, D., Ji, W., Peng, Y., Ji, H., \& Gao, J. (2020b). Evaluation of flavor improvement in Antarctic krill defluoridated hydrolysate by Maillard reaction using sensory analysis, E-nose, and GC-MS. Journal of Aquatic Food Product Technology, 29(3), 279-292. http://dx.doi.or g/10.1080/10498850.2020.1723764.

Zhang, H., Pu, D., Sun, B., Ren, F., Zhang, Y., \& Chen, H. (2018). Characterization and comparison of key aroma compounds in raw and dry porcini mushroom (Boletus edulis) by aroma extract dilution analysis, quantitation and aroma recombination experiments. Food Chemistry, 258, 260-268. http://dx.doi.org/10.1016/j. foodchem.2018.03.056. PMid:29655732.

Zhang, Y., Ma, X., \& Dai, Z. (2019). Comparison of nonvolatile and volatile compounds in raw, cooked, and canned yellow fin tuna (Thunnus albacores). Journal of Food Processing and Preservation, 43(10), e14111. http://dx.doi.org/10.1111/jfpp.14111.

Zhu, W., Luan, H., Bu, Y., Li, X., Li, J., \& Ji, G. (2019). Flavor characteristics of shrimp sauces with different fermentation and storage time. Lebensmittel-Wissenschaft + Technologie, 110, 142-151. http://dx.doi. org/10.1016/j.lwt.2019.04.091.

Zielinski, A. A. F., Alberti, A., Bona, E., Bortolini, D. G., Benvenutti, L., Bach, F., Demiate, I. M., \& Nogueira, A. (2020). A multivariate approach to differentiate yerba mate (Ilex paraguariensis) commercialized in the southern Brazil on the basis of phenolics, methylxanthines and in vitro antioxidant activity. Food Science and Technology, 40(3), 645-652. http://dx.doi.org/10.1590/fst.15919. 